\title{
A facile tandem synthesis of $\alpha$-benzyl benzimidazole acetonitriles
}

\author{
P. K. Dubey, ${ }^{*}$ P. V. V. Prasada Reddy, and K. Srinivas \\ Department of Chemistry, College of Eng., J N T University, Kukatpally, \\ Hyderabad, (A.P.). -500 085, India \\ E-mail:pvvpr@rediffmail.com
}

\begin{abstract}
An efficient and convenient tandem method for the synthesis of $\alpha$-benzylbenzimi dazoleacetonitriles has been developed. In this method, a benzimidazoleacetonitrile 1 was condensed with an aromatic aldehyde in the presence of basic alumina under solvent-free conditions by simple physical grinding of reactants using a mortar and pestle. Subsequent reduction of the in situ formed acrylonitrile derivative with $\mathrm{NaBH}_{4}$ in $95 \%$ ethanol at room temperature gives the corresponding 2-(1H-benzimidazol-2-yl)-3-aryl-propionitrile $\mathbf{3}$ by regiospecific reduction of the double bond.
\end{abstract}

Keywords: Benzimidazoleacetonitriles, aromatic aldehydes, basic alumina, solvent-free, $\mathrm{NaBH}_{4}$

\section{Introduction}

In recent years considerable attention has been paid to the reactions done under solvent-free conditions ${ }^{1,2} . \mathrm{NaBH}_{4}$ is a frequently used hydride in reduction processes and it is known to reduce polar carbonyl groups such as aldehydes and ketones ${ }^{3}$ and esters ${ }^{4}$. It is also known to reduce, selectively, carbonyl groups in $\alpha, \beta$-unsaturated ketones ${ }^{5}$ and nitriles under certain conditions ${ }^{6}$.

Benzimidazole derivatives are useful intermediates/subunits for the development of molecules of pharmaceutical or biological interest ${ }^{7}$ and substituted benzimidazole derivatives have found applications in diverse therapeutic areas such as anti-ulcer agents, anti-cancer agents, and anthelmintic species to name just a few ${ }^{8,9}$. The widespread interest in benzimidazolecontaining systems has promoted extensive studies of their syntheses. Substituted-2-( $\alpha-$ cyanostyryl)benzimidazoles are useful intermediates for the syntheses of fused heterocyclic ring systems ${ }^{10}$.

In continuation of our earlier work ${ }^{11,12}$ on the synthesis of new benzimidazole derivatives with potential biological activity, we have been interested in the synthesis of $2-(\alpha-$ 
cyanostyryl)benzimidazoles and their further reactions with various reagents. The results of our studies in this direction are presented in this communication.

\section{Results and Discussion}

Literature reports ${ }^{13,14}$ indicate that the Knoevenagel condensation of 1-alkyl-2-(1Hbenzimidazol-2-yl)acetonitriles 1 with aromatic aldehydes has been carried out with pyridine, piperidine, piperidine acetate, acetic acid, anhydrous $\mathrm{ZnCl}_{2}$ and $\mathrm{NaOH}$ in methanol as catalysts.

In our earlier communication ${ }^{14}$, we reported that the condensation of $\mathbf{1}$ with aromatic aldehydes in methanolic $\mathrm{NaOH}$ solution gave isolated 1-alkyl-2-(1H-benzimidazol-2-yl)-3arylacrylonitrile 2, which, by subsequent reduction with $\mathrm{NaBH}_{4}$ in ethanol, resulted in the formation of $\mathbf{3}$ in $74-82 \%$ yields. Now we wish to report a more selective method for the synthesis of monobenzylated derivatives of benzimidazole acetonitrile following a two-reaction sequence. The first step is a Knoevenagel condensation between benzimidazole acetonitriles and aromatic aldehydes under solvent-free conditions in the presence of basic alumina. The intermediate 2-( $\alpha$-cyanostyryl)benzimidazoles $\mathbf{2}$ are then reduced in the second step to afford the desired $\alpha$-benzylated benzimidazole acetonitrile derivatives in a tandem method.

Thus, condensation of 2-(1H-benzimidazol-2-yl)acetonitrile ${ }^{15} \mathbf{1 a}$ with $p$-tolualdehyde in the presence of basic alumina under solvent-free conditions was carried out by simple physical grinding using a mortar and pestle at room temperature for 10-15 min. The progress of the reaction was monitored by TLC analysis of the reaction mixture for the disappearance of 1a using hexane and ethyl acetate (8:2) as eluent. Upon completion of the first step, the crude product was transferred into a round bottom flask and charged with $95 \%$ ethanol and the reaction mixture was cooled to $0^{\circ} \mathrm{C}$ before introducing the $\mathrm{NaBH}_{4}$. After complete addition of $\mathrm{NaBH}_{4}$, slowly the reaction mixture was brought to room temperature, maintained at room temperature for 2-2.5 h, followed by an aqueous work-up to yield, 2-(1H-benzimidazol-2-yl)-3tolylpropionitrile $\mathbf{3 a}$, by reduction of the double bond. The nitrile group remained totally unaffected under these conditions.

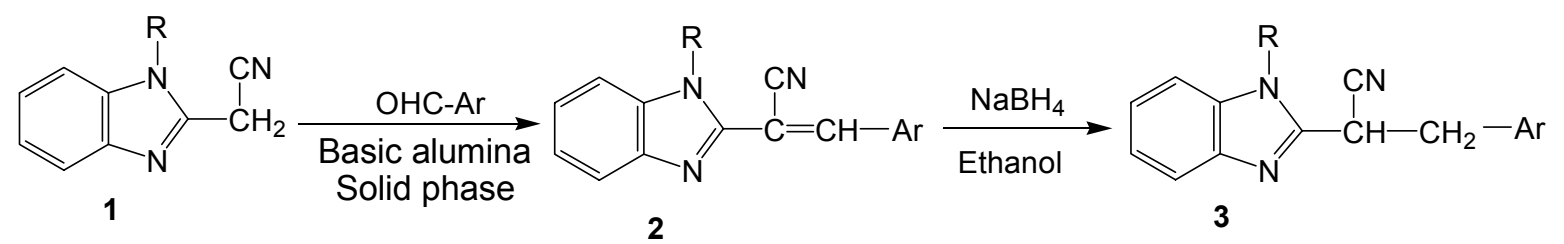

\section{Scheme 1}

The above reaction has been found to be a general one and has been extended to other 1alkyl-2-(1H-benzimidazol-2-yl)acetonitriles $1\left(\mathrm{R}=\mathrm{H} \& \mathrm{CH}_{3}\right)$ (Table 1) with aromatic aldehydes, 
followed by reduction with $\mathrm{NaBH}_{4}$. The products thus obtained were assigned structure 3 on the basis of comparison of their spectral and analytical data with those reported ${ }^{14}$.

It may be mentioned here that Robert et al. ${ }^{16}$ first used this method for preparing mono alkylated derivatives of malononitrile by condensation of malononitrile with carbonyl compounds in the absence of alumina. In our work, it was found that condensation of 1 with aromatic aldehydes did not take place when the reaction between the two was attempted in the solid phase in the absence of alumina.

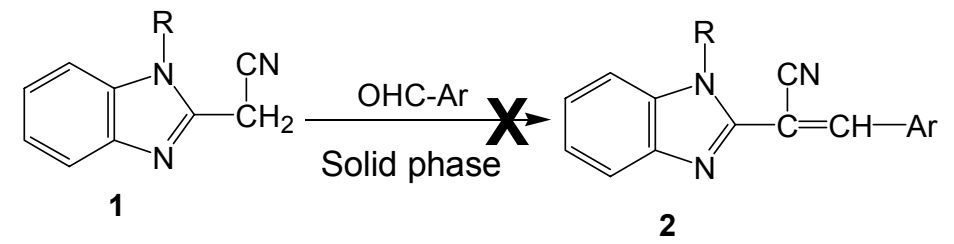

Table 1. Representation of compounds 1- 3 (R \& Ar)

\begin{tabular}{|c|c|c|c|}
\hline Entry & Substrate & $\mathrm{Ar}$ & Product \\
\hline $\mathrm{a}$ & 1a $(\mathbf{R}=\mathrm{H})$ & $-\mathrm{C}_{6} \mathrm{H}_{4} \mathrm{CH}_{3}-p$ & $\mathbf{3 a}$ \\
\hline $\mathrm{b}$ & $1 \mathbf{b}(\mathbf{R}=\mathrm{H})$ & $-\mathrm{C}_{6} \mathrm{H}_{5}$ & $3 \mathbf{b}$ \\
\hline $\mathrm{c}$ & $\mathbf{1 c}(\mathbf{R}=\mathrm{H})$ & $-\mathrm{C}_{6} \mathrm{H}_{4} \mathrm{OCH}_{3}-p$ & $3 c$ \\
\hline $\mathrm{d}$ & $\mathbf{1 d}(\mathbf{R}=\mathrm{H})$ & $-\mathrm{C}_{6} \mathrm{H}_{4} \mathrm{~N}\left(\mathrm{CH}_{3}\right)_{2}-p$ & 3d \\
\hline $\mathrm{e}$ & 1e $(R=H)$ & $-\mathrm{C}_{6} \mathrm{H}_{3}\left(\mathrm{OCH}_{3}\right)-m-\left(\mathrm{OCH}_{3}\right)-p$ & $3 e$ \\
\hline $\mathrm{f}$ & If $(\mathbf{R}=\mathrm{H})$ & $-\mathrm{C}_{6} \mathrm{H}_{4} \mathrm{Cl}-p$ & 3f \\
\hline $\mathrm{g}$ & $\lg (\mathbf{R}=\mathrm{H})$ & $-\mathrm{C}_{6} \mathrm{H}_{4} \mathrm{~F}-p$ & $3 g$ \\
\hline $\mathrm{h}$ & $\mathbf{1 h}\left(\mathbf{R}=\mathrm{CH}_{3}\right)$ & $-\mathrm{C}_{6} \mathrm{H}_{4} \mathrm{CH}_{3}-p$ & $3 \mathbf{h}$ \\
\hline $\mathrm{i}$ & $\mathbf{1 i}\left(\mathbf{R}=\mathrm{CH}_{3}\right)$ & $-\mathrm{C}_{6} \mathrm{H}_{5}$ & $3 \mathbf{i}$ \\
\hline $\mathrm{j}$ & $\mathbf{1 j}\left(\mathbf{R}=\mathrm{CH}_{3}\right)$ & $-\mathrm{C}_{6} \mathrm{H}_{4} \mathrm{OCH}_{3}-p$ & $3 \mathbf{j}$ \\
\hline $\mathrm{k}$ & $1 \mathbf{k}\left(\mathbf{R}=\mathrm{CH}_{3}\right)$ & $-\mathrm{C}_{6} \mathrm{H}_{4} \mathrm{~N}\left(\mathrm{CH}_{3}\right)_{2}-p$ & $3 \mathbf{k}$ \\
\hline 1 & $11\left(\mathbf{R}=\mathrm{CH}_{3}\right)$ & $-\mathrm{C}_{6} \mathrm{H}_{3}\left(\mathrm{OCH}_{3}\right)-m-\left(\mathrm{OCH}_{3}\right)-p$ & 31 \\
\hline
\end{tabular}

When, the reaction of 2-styrylbenzimidazole ${ }^{17} 4\left(\mathrm{R}=\mathrm{H}\right.$ or $\left.\mathrm{CH}_{3}\right)(\mathrm{Scheme}-2)$ with $\mathrm{NaBH}_{4}$ in $95 \%$ ethanol at room temperature was attempted, it led to the recovery of starting material. However, shaking a solution of 4 with $\mathrm{H}_{2}$ gas over $\mathrm{Pd}-\mathrm{C}$ at room temperature gave the corresponding reduced product 5 involving saturation of the double bond. Based on the above findings, it may be inferred that the $\mathrm{C}=\mathrm{C}$ is reduced selectively by $\mathrm{NaBH}_{4}$ when it is in conjugation with an electron withdrawing group like a nitrile, due to the increase in the polarization of the double bond. 


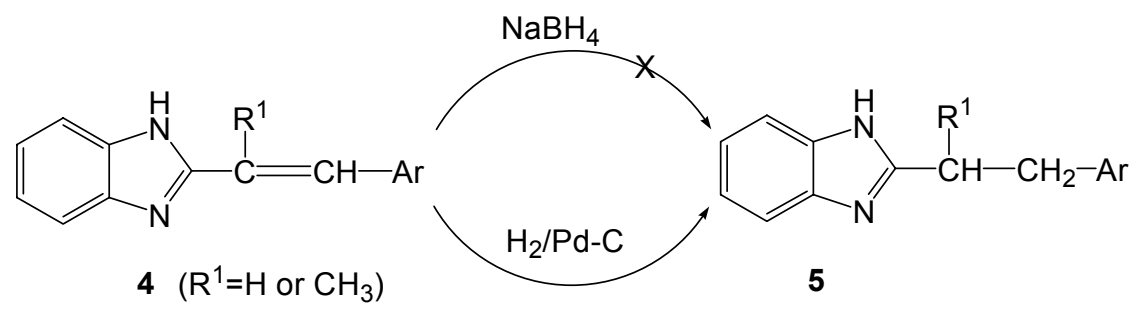

\section{Scheme 2}

In conclusion, we have developed a simple, novel, efficient and highly selective preparation of $\alpha$-benzylated benzimidazoleacetonitriles by using basic alumina and $\mathrm{NaBH}_{4}$ in a tandem method.

\section{Experimental Section}

General Procedures. Melting points are uncorrected and were determined in open capillary tubes in a sulphuric acid bath. TLC was performed on silica gel-G and visualization was done using iodine or UV-light. IR spectra were recorded with a Perkin-Elmer 1000 instrument in the $\mathrm{KBr}$ phase. ${ }^{1} \mathrm{H}-\mathrm{NMR}$ spectra were obtained on a VARIAN $200 \mathrm{MHz}$ instrument and Mass spectra were recorded on an Agilent-LC-MS instrument giving only $\mathrm{M}^{+}$. values using the $\mathrm{Q}+1$ mode.

\section{General experimental method}

A mixture of $1(10 \mathrm{mmol})$, basic alumina $(1.0 \mathrm{~g})$ and aromatic aldehydes $(10 \mathrm{mmol})$ was taken in a mortar and ground with a pestle. The progress of the reaction was monitored by TLC. After complete depletion of the starting material, the crude mass was transferred into a round bottom flask for reduction. To the crude mass, ethanol $(50 \mathrm{~mL})$ was added and the mixture was cooled to $0^{\circ} \mathrm{C}$ in a salt-ice bath. $\mathrm{NaBH}_{4}(0.378 \mathrm{~g}, 10 \mathrm{mmol})$ was then introduced in one portion and the reaction mass was brought to RT slowly and stirred for 2.0-2.5 h. After completion of the reaction, the ethanol (20-25 $\mathrm{mL})$ was distilled off and the reaction mixture was poured into icecold water $(100 \mathrm{~mL})$ and extracted into ethyl acetate $(2 \times 25 \mathrm{~mL})$. The combined organic layers were dried with anhyd. $\mathrm{Na}_{2} \mathrm{SO}_{4}$ and evaporated to give pure product 3 without any further purification.

2-(1H-Benzimidazol-2-yl)-3-p-tolylpropionitrile (3a). Yield: 94\%, mp: 208-10 C, IR (KBr) $\mathrm{cm}^{-1}: 3084(-\mathrm{NH}), 2254(-\mathrm{CN}) ;{ }^{1} \mathrm{H}$ NMR (200MHz, DMSO-d $): \delta 2.24\left(\mathrm{~s}, 3 \mathrm{H},-\mathrm{CH}_{3}\right), 3.33(\mathrm{~m}$, $\left.2 \mathrm{H},-\mathrm{CH}_{2}-\right), 4.85(\mathrm{t}, 1 \mathrm{H},-\mathrm{CH}-), 7.08-7.60(\mathrm{~m}, 8 \mathrm{H}, \mathrm{Ar}-\mathrm{H}), 12.70\left(\mathrm{~s}, 1 \mathrm{H},-\mathrm{NH}-, \mathrm{D}_{2} \mathrm{O}\right.$ exchangeable). $\mathrm{M} / \mathrm{z}\left(\mathrm{M}^{+}+1\right)$ : 262. Anal. Calcd. for $\left(\mathrm{C}_{17} \mathrm{H}_{15} \mathrm{~N}_{3}\right)$ requires: $\mathrm{C}, 78.13 ; \mathrm{H}, 5.79 ; \mathrm{N}$, 16.08; Found: 78.10; H, 5.75; N, $15.98 \%$.

2-(1H-Benzimidazol-2-yl)-3-phenylpropionitrile (3b). Yield: $92 \%, \mathrm{mp}: 176-78^{\circ} \mathrm{C}, \mathrm{IR}(\mathrm{KBr})$ $\mathrm{cm}^{-1}: 3057(-\mathrm{NH}), 2252(-\mathrm{CN}) .{ }^{1} \mathrm{H}$ NMR (200MHz, DMSO-d 6$): \delta 3.35\left(\mathrm{~m}, 2 \mathrm{H},-\mathrm{CH}_{2}-\right), 4.90(\mathrm{t}$, 
1H,-CH-), 7.12-7.63(m, 9H, Ar-H), 12.70(s, 1H, D $\mathrm{O}_{2} \mathrm{O}$ exchangeable -NH). M/z (M $\left.{ }^{+}+1\right): 248$. Anal. Calcd. for $\left(\mathrm{C}_{16} \mathrm{H}_{13} \mathrm{~N}_{3}\right)$ requires: C, 77.71; H, 5.30; N. 16.99;

Found: C, 77.72; H, 5.28; 16.96\%.

2-(1H-Benzimidazol-2-yl)-3-(4-methoxyphenyl)propionitrile (3c). Yield: 95\%, mp: 196-98 $\mathrm{C}$, IR (KBr) cm ${ }^{-1}: 3081(-\mathrm{NH}), 2242(-\mathrm{CN}) .{ }^{1} \mathrm{H}$ NMR (200MHz, DMSO-d 6 ): $\delta .3 .33\left(\mathrm{~m}, 2 \mathrm{H},-\mathrm{CH}_{2}-\right)$, 3.70(s, 3H, $\left.-\mathrm{OCH}_{3}\right), 4.83(\mathrm{t}, 1 \mathrm{H},-\mathrm{CH}-)$ 6.8-7.6(m, 8H, Ar-H), 12.69(s, 1H, $\mathrm{D}_{2} \mathrm{O}$ exchangeable $\mathrm{NH}) . \quad \mathrm{M} / \mathrm{z}\left(\mathrm{M}^{+}+1\right): 278$. Anal. Calcd. for $\left(\mathrm{C}_{17} \mathrm{H}_{15} \mathrm{~N}_{3} \mathrm{O}\right)$ requires: $\mathrm{C}, 73.63 ; \mathrm{H}, 5.45 ; \mathrm{N}, 15.15$; Found: C, 73.61; H, 5.47; N, 15.14\%.

2-(1H-Benzimidazol-2-yl)-3-(4-dimethylaminophenyl)propionitrile (3d). Yield: 90\%, mp: 206-08 ${ }^{\circ} \mathrm{C}$, IR (KBr) cm ${ }^{-1}: 3081(-\mathrm{NH}), 2245(-\mathrm{CN}) .{ }^{1} \mathrm{H}$ NMR $\left(200 \mathrm{MHz}, \mathrm{DMSO}-\mathrm{d}_{6}\right): \delta 2.83(\mathrm{~s}$, $\left.6 \mathrm{H},-\mathrm{N}\left(\mathrm{CH}_{3}\right)_{2}\right), 3.29\left(\mathrm{~m}, 2 \mathrm{H},-\mathrm{CH}_{2}-\right)$, 4.76(t, 1H,-CH-), 6.6-7.6(m, 8H, Ar-H), 12.68(s, 1H, D $\mathrm{O}^{\mathrm{O}}$ exchangeable $-\mathrm{NH}) . \mathrm{M} / \mathrm{z}\left(\mathrm{M}^{+}+1\right)$ : 291. Anal. Calcd. for $\left(\mathrm{C}_{18} \mathrm{H}_{18} \mathrm{~N}_{4}\right)$ requires: $\mathrm{C}, 74.46 ; \mathrm{H}, 6.25$; N, 19.30; Found: C, 74.42; H, 6.24; N, 19.26\%.

2-(1H-Benzimidazol-2-yl)-3-(3,4-dimethoxyphenyl)propionitrile (3e). Yield: 92\%, mp: 186$88^{\circ} \mathrm{C}$, IR (KBr) cm ${ }^{-1}: 3075(-\mathrm{NH}), 2238(-\mathrm{CN}) .{ }^{1} \mathrm{H}$ NMR $\left(200 \mathrm{MHz}, \mathrm{DMSO}-\mathrm{d}_{6}\right): \delta 3.33(\mathrm{~m}, 2 \mathrm{H}$, $\left.\mathrm{CH}_{2}-\right), 3.59\left(\mathrm{~s}, 3 \mathrm{H},-\mathrm{OCH}_{3}\right), 3.69\left(\mathrm{~s}, 3 \mathrm{H},-\mathrm{OCH}_{3}\right), 4.85(\mathrm{t}, 1 \mathrm{H},-\mathrm{CH}-), 6.78-7.62(\mathrm{~m}, 7 \mathrm{H}, \mathrm{Ar}-\mathrm{H})$, 12.68(s, $1 \mathrm{H}, \mathrm{D}_{2} \mathrm{O}$ exchangeable-NH). $\mathrm{M} / \mathrm{z}\left(\mathrm{M}^{+}+1\right)$ : 308 . Anal. Calcd. for $\left(\mathrm{C}_{18} \mathrm{H}_{17} \mathrm{~N}_{3} \mathrm{O}_{2}\right)$ requires: C, 70.34; H, 5.58; N, 13.67; Found: 70.36; H, 5.56; 13.63\%.

2-(1H-Benzimidazol-2-yl)-3-(4-chlorophenyl)propionitrile (3f). Yield: 90\%, mp: 206-08 ${ }^{\circ} \mathrm{C}$, IR (KBr) cm ${ }^{-1}: 3024$ (-NH), 2249 (-CN). ${ }^{1} \mathrm{H}$ NMR (200MHz, DMSO-d 6 ): $\delta 3.43$ (m, 2H, $-\mathrm{CH}_{2^{-}}$), 4.85 (t, 1H, -CH-), 7.12-7.62 (m, 8H, Ar-H), 12.7 (s, 1H, $\mathrm{D}_{2} \mathrm{O}$ exchangeable-NH). $\mathrm{M} / \mathrm{z}\left(\mathrm{M}^{+}+1\right)$ : 282. Anal. Calcd. for $\left(\mathrm{C}_{16} \mathrm{H}_{12} \mathrm{ClN}_{3}\right)$ requires: $\mathrm{C}, 68.21$; H, 4.29; N,14.91; Found: $\mathrm{C}, 68.20$; H, 4.26, N, 14.93\%.

2-(1H-Benzimidazol-2-yl)-3-(4-fluorophenyl)propionitrile (3g). Yield: 88\%, mp: 170-72 $\mathrm{C}$, IR (KBr) cm ${ }^{-1}: 3085(-\mathrm{NH}), 2252(-\mathrm{CN}) .{ }^{1} \mathrm{H}$ NMR $\left(200 \mathrm{MHz}, \mathrm{DMSO}-\mathrm{d}_{6}\right): \delta 3.30\left(\mathrm{~m}, 2 \mathrm{H},-\mathrm{CH}_{2^{-}}\right)$, 4.9 (t, 1H, -CH-), 7.12-7.7 (m, 8H, Ar-H), 12.65 (s, 1H, $\mathrm{D}_{2} \mathrm{O}$ exchangeable-NH). M/z (M $\left.{ }^{+}+1\right)$ : 266. Anal. Calcd. for $\left(\mathrm{C}_{16} \mathrm{H}_{12} \mathrm{FN}_{3}\right)$ requires: $\mathrm{C}$, 72.44; $\mathrm{H}, 4.56 ; \mathrm{N}, 15.84$; Found: $\mathrm{C}, 72.40$; $\mathrm{H}$, $4.58 ; \mathrm{N}, 15.82 \%$.

2-(1-Methyl-1H-benzimidazol-2-yl)-3-p-tolylpropionitrile (3h). Yield: 96\%, mp: 134-36 ${ }^{\circ} \mathrm{C}$, IR (KBr) cm ${ }^{-1}: 2247(-\mathrm{CN}) .{ }^{1} \mathrm{H}$ NMR $\left(200 \mathrm{MHz}, \mathrm{CDCl}_{3}\right): \delta 2.33\left(\mathrm{~s}, 3 \mathrm{H},-\mathrm{CH}_{3}\right), 3.50\left(\mathrm{~m}, 2 \mathrm{H},-\mathrm{CH}_{2-}\right.$ ), 3.59(s, 3H, $\left.-\mathrm{NCH}_{3}\right), 4.35(\mathrm{t}, 1 \mathrm{H},-\mathrm{CH}-), 7.12-8.0(\mathrm{~m}, 8 \mathrm{H}, \mathrm{Ar}-\mathrm{H}) . \mathrm{M} / \mathrm{z}\left(\mathrm{M}^{+}+1\right): 276$. Anal. Calcd. for $\left(\mathrm{C}_{18} \mathrm{H}_{17} \mathrm{~N}_{3}\right)$ requires: $\mathrm{C}, 78.52 ; \mathrm{H}, 6.22 ; \mathrm{N}, 15.26$; Found: $\mathrm{C}, 78.49 ; \mathrm{H}, 6.21 ; 15.28 \%$.

2-(1-Methyl-1H-benzimidazol-2-yl)-3-phenylpropionitrile (3i). Yield: 92\%, mp: 142-44 C, IR $(\mathrm{KBr}) \mathrm{cm}^{-1}: 2245(-\mathrm{CN}) .{ }^{1} \mathrm{H} \mathrm{NMR}\left(200 \mathrm{MHz}, \mathrm{CDCl}_{3}\right): \delta 3.55\left(\mathrm{~m}, 2 \mathrm{H},-\mathrm{CH}_{2}-\right), 3.58\left(\mathrm{~s}, 3 \mathrm{H},-\mathrm{NCH}_{3}\right)$, 4.36(t, 1H, -CH-), 7.21-7.82(m, 9H, Ar-H). M/z (M $\left.{ }_{+}^{+}+1\right)$ : 262. Anal. Calcd. for $\left(\mathrm{C}_{17} \mathrm{H}_{15} \mathrm{~N}_{3}\right)$ requires: C, 78.13; H, 5.79; N, 16.08; Found: C, 78.10; H, 5.80; N, 16.02\%.

3-(4-Methoxyphenyl)-2-(1-methyl-1H-benzimidazol-2-yl)propionitrile (3j). Yield: 95\%, mp: $148-50^{\circ} \mathrm{C}$, IR $(\mathrm{KBr}) \mathrm{cm}^{-1}: 2247(-\mathrm{CN}) .{ }^{1} \mathrm{H} \mathrm{NMR}\left(200 \mathrm{MHz}, \mathrm{CDCl}_{3}\right): \delta 3.47\left(\mathrm{~m}, 2 \mathrm{H},-\mathrm{CH}_{2}-\right)$, $3.50\left(\mathrm{~s}, 3 \mathrm{H},-\mathrm{NCH}_{3}\right), 3.78\left(\mathrm{~s}, 3 \mathrm{H},-\mathrm{OCH}_{3}\right), 4.32(\mathrm{t}, 1 \mathrm{H},-\mathrm{CH}-), 6.82-7.70(\mathrm{~m}, 8 \mathrm{H}, \mathrm{Ar}-\mathrm{H}) . \mathrm{M} / \mathrm{z}(\mathrm{M}$ 
$\left.{ }_{+}^{+} 1\right)$ : 292. Anal. Calcd. for $\left(\mathrm{C}_{18} \mathrm{H}_{17} \mathrm{~N}_{3} \mathrm{O}\right)$ requires: C, 74.20; H, 5.88; N, 14.42; Found: C, 74.18; H, 5.85; N, 14.40\%.

3-(4-Dimethylaminophenyl)-2-(1-methyl-1H-benzimidazol-2-yl)propionitrile (3k). Yield: 91\%, mp: $120-22^{\circ} \mathrm{C}$, IR (KBr) cm ${ }^{-1}: 2244(-\mathrm{CN}) .{ }^{1} \mathrm{H}$ NMR $\left(200 \mathrm{MHz}, \mathrm{CDCl}_{3}\right): \delta 2.9(\mathrm{~s}, 6 \mathrm{H},-\mathrm{N}$ $\left.\left(\mathrm{CH}_{3}\right)_{2}\right), 3.42\left(\mathrm{~m}, 2 \mathrm{H},-\mathrm{CH}_{2}-\right), 3.50\left(\mathrm{~s}, 3 \mathrm{H},-\mathrm{NCH}_{3}\right), 4.3(\mathrm{t}, 1 \mathrm{H},-\mathrm{CH}-), 6.6-7.8(\mathrm{~m}, 8 \mathrm{H}) . \mathrm{M} / \mathrm{z}(\mathrm{M}$ $\left.{ }^{+}+1\right)$ : 305. Anal. Calcd. for $\left(\mathrm{C}_{19} \mathrm{H}_{20} \mathrm{~N}_{4}\right)$ requires: $\mathrm{C}, 74.97 ; \mathrm{H}, 6.62 ; \mathrm{N}, 18.41$; Found: $\mathrm{C}, 74.96 ; \mathrm{H}$, $6.60 ; \mathrm{N}, 18.44 \%$.

3-(3,4-Dimethoxyphenyl)-2-(1-methyl-1H-benzimidazol-2-yl)propionitrile (3I). Yield: 90\%, mp: $128-30^{\circ} \mathrm{C}$, IR $(\mathrm{KBr}) \mathrm{cm}^{-1}: 2246(-\mathrm{CN}) .{ }^{1} \mathrm{H}$ NMR $\left(200 \mathrm{MHz}, \mathrm{CDCl}_{3}\right): \delta 3.5\left(\mathrm{~s}, 3 \mathrm{H},-\mathrm{OCH}_{3}\right)$, $3.6\left(\mathrm{~s}, 3 \mathrm{H},-\mathrm{OCH}_{3}\right), 3.8\left(\mathrm{~m}, 2 \mathrm{H},-\mathrm{CH}_{2}-\right), 3.82\left(\mathrm{~s}, 3 \mathrm{H},-\mathrm{NCH}_{3}\right), 4.3(\mathrm{t}, 1 \mathrm{H},-\mathrm{CH}-), 6.6-7.8(\mathrm{~m}, 8 \mathrm{H}, \mathrm{Ar}-$ H). $\mathrm{M} / \mathrm{z}\left(\mathrm{M}^{+}+1\right)$ : 322. Anal. Calcd. for $\left(\mathrm{C}_{19} \mathrm{H}_{19} \mathrm{~N}_{3} \mathrm{O}_{2}\right)$ requires: $\mathrm{C}, 71.01 ; \mathrm{H}, 5.96 ; \mathrm{N}, 13.08$; Found: C, 70.98; H, 5.98, N, 13.06\%.

\section{Acknowledgements}

The authors are highly indebted to the University Grants Commission, Govt. of India, New Delhi for financial support of this work.

\section{References}

1. Merzger, J. D. Angew. Chem. Int Ed. 1998, 37, 2975.

2. Tanaka, K.; Toda, F. Chem. Rev. 2000, 100, 1025.

3. (a) Chaikin, S. W.; Brown, W. G. J. Am. Chem. Soc. 1949, 71, 122. (b) Nishimura, T.; Nakajima, M.; Madea, Y.; Uemura, S.; Takekuma, S.; Takekuma, H.; Yoshida, Z. Bull. Chem. Soc. Jpn. 2004, 77, 2047.

4. (a) Prasad, A. B. S.; Kanth, J. V. B.; Periassamy, M. Tetrahedron 1992, 48, 4623. (b) Nubia, B.; Jorge, C. S. da. C.; Jorge de, S. M.; Pedro, S. M. de. O.; Marcus, V. N. De. S. Tetrahedron Lett. 2004, 45, 6021.

5. Singh, J.; Kaur, J.; Bhalla, A.; Kad, G. L. Synth. Commun. 2003, 33, 191. (b) Zhou, Q.; Tang, Y.; Wang, L.; Zhao, G.; Zhou, Q.; Tang, C. Synthesis 2004, 217.

6. (a) Akabori, S.; Takanohashi, Y. J. Chem. Soc., Perkin Trans. 1 1991, 479. (b) Mauger, J.; Robert, A. J. Chem. Soc., Chem. Commun. 1986, 395.

7. (a) Erhardt, P.W. J. Med. Chem. 1987, 30, 231. (b) Tomczuk, B. E.; Taylor, C. R., Jr.; Moses, L. M.; Sutherland, D. B.; Lo, Y. S.; Johnson, D. N.; Kinnier, W. B.; Kilpatrick, B. F. J. Med. Chem. 1991, 34, 2993. (c) Spasov, A. A.; Yozhitsa, I. N.; Bugaeva, L. I.; Anisimova, V. A. Pharm. Chem. J. 1999, 33, 232. (d) Preston, P. N. Chem. Heterocycl. Compd. 1980, 40, 31. (e) Zimmer, C.; Wahnert, U. Prog. Biophys. Mol. Biol. 1986, 47, 31. (f) Gravatt, G. L.; Baguley, B. C.; Wilson, W, R.; Denny, W. A. J. Med. Chem. 1994, 37, 
4338. (g) Soderlind, K.-J.; Gorodetsky, B.; Singh, A. K.; Bachur, N.; Miller, G. G.; Lown, J. W. Anti-cancer Drug Design. 1999, 14, 19. (h) Grimmet, M. R. In Comprehensive Heterocyclic Chemistry; Katritzky, A. R.; Rees, C. W., Eds., 1984; Vol. 5, p 457.

8. As inhibitors of DNA topoisomerases: (a) Kim, J. S.; Gatto, B.; Yu, C.; Liu, A.; Liu, L. F.; LaVoie, E. J. J. Med. Chem. 1996, 39, 992. (b) Chen, A. Y.; Yu, C.; Gatto, B.; Liu, L. F. Proc. Natl. Acad. Sci. U. S. A. 1993, 90, 8131. (c) Woynarowski, J. M.; McHugh, M. M.; Sigmud, R. D.; Beerman, T. A. Mol. Pharmacol. 1989, 35, 177.

9. As HIV-reverse transcriptase inhibitors: Roth, T.; Morningstar, M. L.; Boyer, P. L.; Hughes, S. H.; Buckheit, R. W. Jr.; Michejda, C. J. J. Med. Chem. 1997, 40, 4199.

10. (a) Hammad, M.; Abdelmeguid, S.; EI-Anani, M. M.; Shafik, N. Egypt. J. Chem. 1986, 29(5), 549. (b) Hammad, M. A.; Kamel, M. M.; Abbasi, M. M.; EL-Wassimi, M. T.; Hassan, H. N. A. Pharmazi 1986, 41, 141. (c) Bogdanowicz-Szwed, K.; Czarny, A. J. Pract. Chem. /Chem-Ztg. 1993, 335(3), 279. (d) Alexander, D. T.; Andrey, A. T.; Anton, V. T. Synthesis 2004, 3, 373. (e) Mustapha, R.; Aicha, D.; Jean, P. B.; Jack, H. Tetrahedron Lett. 1994, 35, 4563.

11. Dubey. P. K.; Reddy. P. V. V. P.; Srinivas. K. Synth.. Commun. 2007, 37, 1675.

12. Dubey. P. K.; Reddy. P. V. V. P.; Srinivas, K. Indian J. Chem. 2007, 46B, 488.

13. (a) Hammad. M.; Abdel. M. S.; EI-Anani. M. M.; Shafik. N. Egypt J. Chem. 1986, 29(5), 549. (b) Yamada. E.; Sato, M.; Sugihara. M.; Ohtake. K. Jpn. Pat., 73 29, 517 (1973); Chem.Abstr. 1974, 80, 146971r. (c) Guenther. D.; Ercke. R. Ger Pat, 2,640,549; Chem. Abstr. 1978, 89, 26026a. (d) Venkataratnam, R. V.; Rao. P. S. Indian. J. Chem. 1993, 32B, 484.

14. Dubey. P. K.; Reddy. P. V. V. P. Synth Commun. 2007, 37, 2259.

15. (a) Day, A. R.; Cope land, R. A. B. J.Am. Chem. Soc. 1943, 65, 1072. (b) Buchi, J.; Zwieky, H.; Aebi, A. Arch. Pharm. 1960, 293, 758. (c) Nicole, V. Bull. Soc. Chim. Fr. 1966, 3989. (d) Norman, J. D. U.S. 1959, 2,918,369; Chem. Abstr. 1960, 54, 9577b.

16. (a) Sammelson. R. E.; Allen. M. J. Synthesis 2005, 4, 543. (b) Dunham, J. C.; Richardson, A. D.; Sammelson. R. E. Synthesis 2006, 4, 680.

17. (a) Dubey, P. K.; Ramesh, K.; Ravi Kumar, C.; Grossert, J. S.; Hooper, D. L. Synth. Commun. 2001, 31, 3439. (b) Ramaiah, K.; Dubey, P. K.; Eswara Rao, D.; Ramanatham, J.; Ramesh, K.; Grossert, J. S.; Hooper, D. L. Indian J. Chem. 2000, 39B, 904. 